\title{
Synthesis and Structural Characteristics of Semi-Synthetic Biopolymer Blends Based on Polyvinyl Alcohol, Starch and Chitosan
}

\author{
A. ElShahawy*, S. I. Badr and, A. A. Shadi
}

Physics Department, Faculty of Science, Mansoura University, Mansoura, Egypt

amalelshahawy83@gmail.com

\begin{abstract}
Structural and optical studies in combination with surface morphology investigations were accomplished for blend sample (70/30)wt\% PVA/Starch in addition to other samples of the same composition containing added pre-calculated chitosan contents via ordinary casting route. All synthesized samples were tested using various experimental techniques including Fourier transform infrared FT-IR, UV-visible spectroscopic measurements and scanning electron microscopic (SEM). Variations in FT-IR, UV/vis. band positions and intensities were used as an evidence for the interaction between constituent partner polymeric matrices. The X-ray diffraction (XRD) experimental data reveled that introduction of chitosan into the polymeric matrices results in a decreasing of percent crystallinity through material. SEM micrographs for samples containing chitosan different concentration revealed small granules and bright spots that attributed to starch. Adding up to $80 \%$ more chitosan caused the cryo-fractured surface to become rougher and more brittle.
\end{abstract}

Keywords: PVA/Starch; chitosan; FT-IR; SEM

Language: English

Date of Publication: 30-07-2018

DOI: $10.24297 /$ jap.v14i2.7477

ISSN: 2347-3487

Volume: 14 Issue: 2

Journal: Journal of Advances in Physics

Website: https://cirworld.com

This work is licensed under a Creative Commons Attribution 4.0 International License. 


\section{Introduction}

In recent years, research fields were expand to new borders concerning low cost and high performance polymeric and semi-polymeric materials for industrial and medical applications. In addition, ecofriendly partners are in focus due to growing environmental awareness. Renewability, recyclability, sustainability, and biodegradability are terms to be focused during preparation processes.

Polyvinyl alcohol (PVA) ranks among the few biodegradable synthetic polymers that are frequently modified owing to their properties. PVA may form hydrogen bonding with water due to the presence of huge polar alcohol groups in their main structure that makes them a water soluble polymer with excellent chemical resistance and good mechanical properties. There have been made efforts to develop PVA functionality and biodegradability through blending processes with various synthetic or natural polymers and additives.

Stach and chitosan belongs to the group of biodegradable, natural polymers applied in practice [1 - 3] Starch a widely used natural polymers represent a class of polysaccharides composed of both linear (amylose) and branched (amylopectin). Starch is a low cost, available, and degradable in water [4].

Chitosan can be considered as the second most abundant natural polymeric matrix prepared by deacetylation process of chitin available in shell fish [5]. Chitosan monomer can be considered as 1, 4 linked 2deoxy-2-aminoglucose. Chitosan polymer and their blend or composites take place in sensing processes, tissue engineering, water treatment and film separation due to their superior antioxidant and antibacterial characteristics [6-8].

This work presents synthesis and structural characteristics of semisynthetic biopolymer blends based on polyvinyl alcohol, starch and chitosan to be used in different human contact applications.

\section{Materials and experimental methods}

\subsection{Chemicals}

In order to achieve biodegradable formulations the following raw materials were used: PVA having polymerization degree in the range $1700-1800$ and $98-99^{\circ}$ of hydrolysis, $12-15 \mathrm{CP}$ viscosity, max. $5 \%$ volatile and $0.398 \mathrm{~g} / \mathrm{cm}^{3}$ density. Wheat starch with $800 \mathrm{~kg} / \mathrm{m}^{3}$ density, $\mathrm{pH}$ (30\%suspension) $6-8$. Chitosan of the deacetylation degree of $75 \%$ and molecular weight $\mathrm{M}=1.5 \times 105 \mathrm{~g} / \mathrm{mol}$. All from Sigma Chemicals

\subsection{Sample preparation}

A stock solution of PVA/starch (70/30)wt/wt\% was prepared via dissolving pre-calculated mass of each polymer separately in a double distilled water for $12 \mathrm{~h}$ vigorously stirred at suitable temperatures. Chitosan was loaded to PVA/starch blend at different concentrations. The obtained mixtures were cast onto petri dishes and dried at ambient temperature. Synthesized membranes were then peeled from dishes and stored in desiccator.

Table 1. Sample code and composition of prepared ternary blends

\begin{tabular}{ccc}
\hline Code Sample & PVA/Starch (70/30) \% & Chitosan \% \\
\hline S1 & $100 \%$ & $0.0 \%$ \\
S2 & $90 \%$ & $10 \%$ \\
S3 & $70 \%$ & $30 \%$ \\
S4 & $60 \%$ & $40 \%$
\end{tabular}




\subsection{Characterization Methods}

FT-IR spectral data of all samples were measured twice with 32 scan in absorption mode with a single beam Nicolet is10 spectrophotometer within the spectral range $4000-400 \mathrm{~cm}^{-1}$ and with $4 \mathrm{~cm}^{-1}$ resolution. Ultraviolet-Visible (UV-vis.) optical absorbance data were carried out in the wavelength range from 200-600 $\mathrm{nm}$ using a (Perkin- Elmer UV-VIS) spectrophotometer. X-ray patterns (XRD) of selected samples were analyzed via (Philips X-Pert MPD) X-ray diffractometer occupied with $\mathrm{Cu}$ Ka source operated at $40 \mathrm{kV}$ and 30 $\mathrm{mA}$ within Bragg angles $2 \theta$ ranging $3-40^{\circ}$. Scanning electron micrograph (SEM) was performed on a spurted gold sample coated with a layer (3.5 nm) using SEM (JEOL-JSM 6100).

\section{Results and discussion}

\subsection{Fourier-transform infrared (FT-IR) spectroscopy}

FTIR spectroscopy was used to identify the actual vibrational groups present in the material structure and to study the interaction between such groups in the polymeric backbone when more than polymer or additives added [9].

The spectra of PVA/ starch blends were studied and compared with the spectra of the PVA/starch that contain different concentrations of chitosan. All spectra are presented in Fig. 1. Peaks due to water in the films occur at $1646 \mathrm{~cm}^{-1}$ and around $3462 \mathrm{~cm}^{-1}$. The later peak overlaps the peak due to the $\mathrm{O}-\mathrm{H}$ stretching of hydroxyl groups [10]. Peaks at 2925, 1420 and 853 and1087 $\mathrm{cm}^{-1}$ are attributed to the $\mathrm{C}-\mathrm{H}$ stretching, $\mathrm{C}-\mathrm{H}$ bending and $\mathrm{C}-\mathrm{O}$ stretching of PVA, respectively.

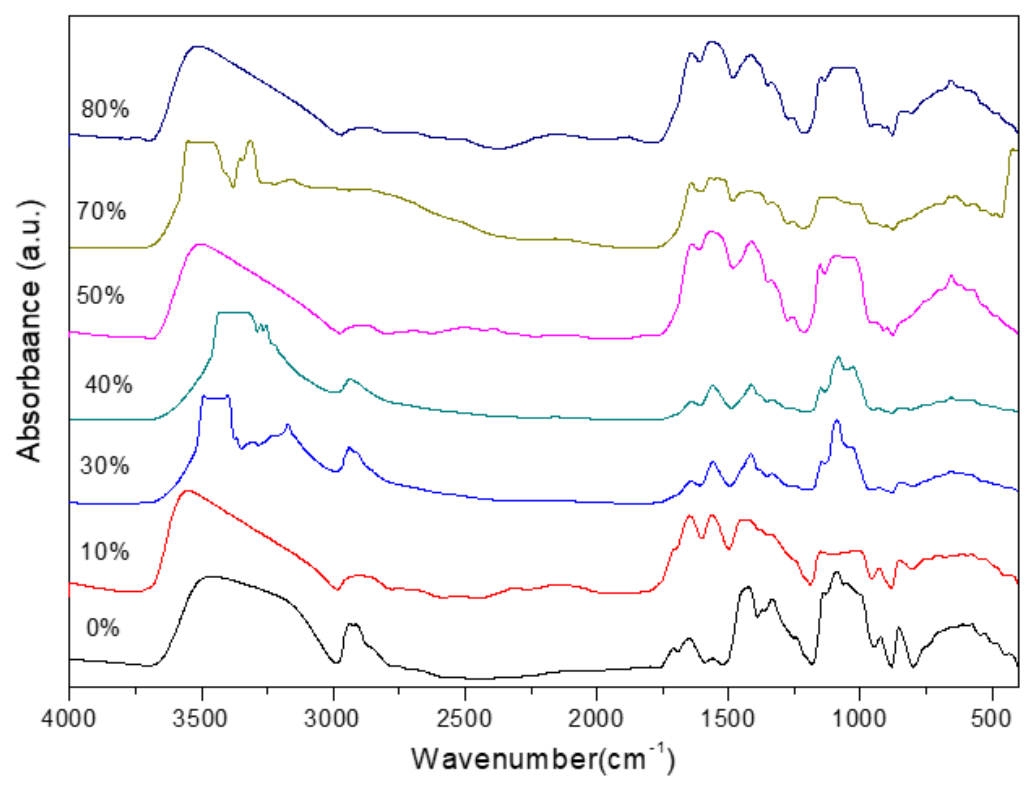

Fig 1: Infrared spectra of PVA/starch blend containing different concentration of chitosan 


\subsection{UV-VIS spectroscopy}

The UV/Vis absorbance spectrum of studied samples recorded in the wavelength range $200-600 \mathrm{~nm}$ are demonstrated in Fig. 2.

Inspection of the recorded spectra reveal presence of a shoulder at $308 \mathrm{~nm}$ attributed usually to the presence of unsaturated carbonyl-groups indicating existence of polynene conjugated double bonds [11]. Observed shoulder intensity varied due to occurrence of isolated carbonyl groups that result in a shift of shoulder position to a higher wavelengths. Such a shifts can be considered as an evidence for complexation and interaction behavior of chitosan with proposed polymeric blend in combination with the observed crystallinity changes attributed to chitosan addition [12].

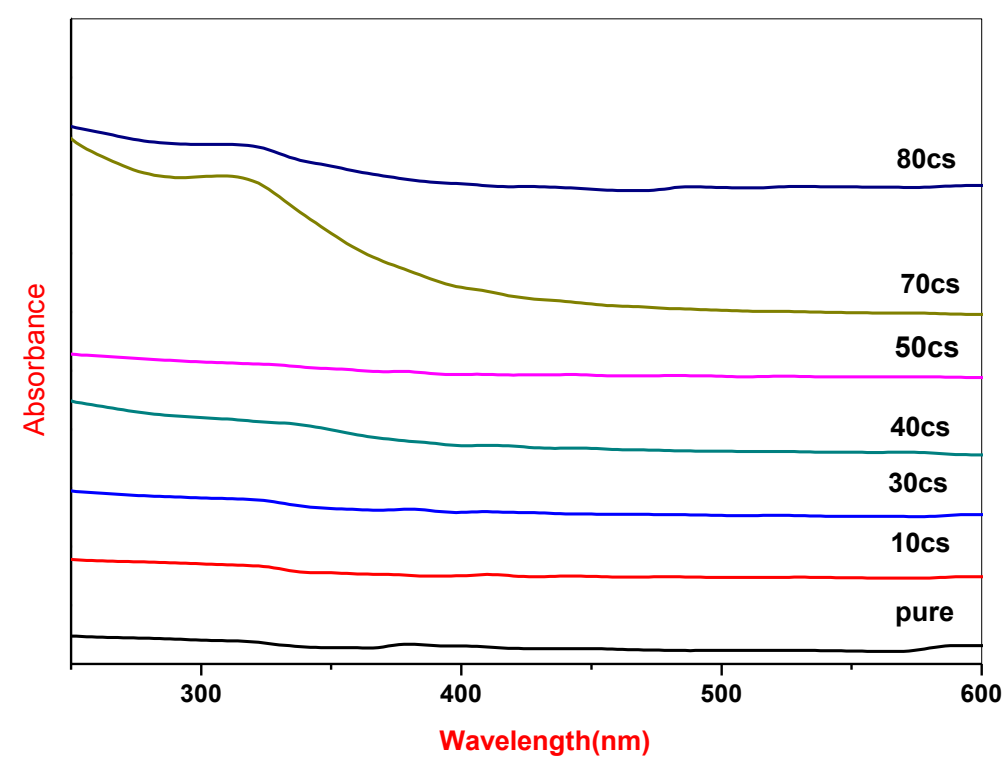

Fig 2: UV-vis spectra for PVA/starch blend containing different concentration of chitosan

\subsubsection{Determination of optical energy gap (Eg):}

Study of electronic transition via optical absorption gives valuable information about band structure. Types of possible electron transitions can be determined by the changes in the absorbed radiation.

A sudden change in the fundamental absorption can be defined as (absorption edge) which accurately used with the absorption coefficient . . . in terms of absorbance $(A)$ and thickness $(d)$ to estimate optical band gap $\left(E_{g}=h c / \lambda\right), \alpha=2.303 \mathrm{~A} / \mathrm{d}$.

Energy of incident photon was reported by different authors $[13,14]$ to be correlated with absorption coefficient through the famous formalism;

$$
(\alpha h v)=C\left(h v-E_{g}\right)^{r} \quad \text { for } h v>E_{g}
$$

where Eg is the optical energy gap and $C$ is an adjustable constant. The power $r$ can take values $1,2,3,1 / 2$ and $3 / 2$ correlated to momentum space and type of transition.

UV/vis. optical spectroscopic measurements and data analysis can be used to estimate optical constants including position of fundemntal edge and optical band gap (Eg). According to Shahada et al [15] based in $r$ values the type of transition can be detected through introduction of what is called Tauc's plots. Extrapolated line of $(\alpha h v)^{2}$ or $(\alpha h v)^{1 / 2}$ versus (hv) can gives accurate values for the optical energy gaps as shown in an exemplified Figure $(3,4)$ 

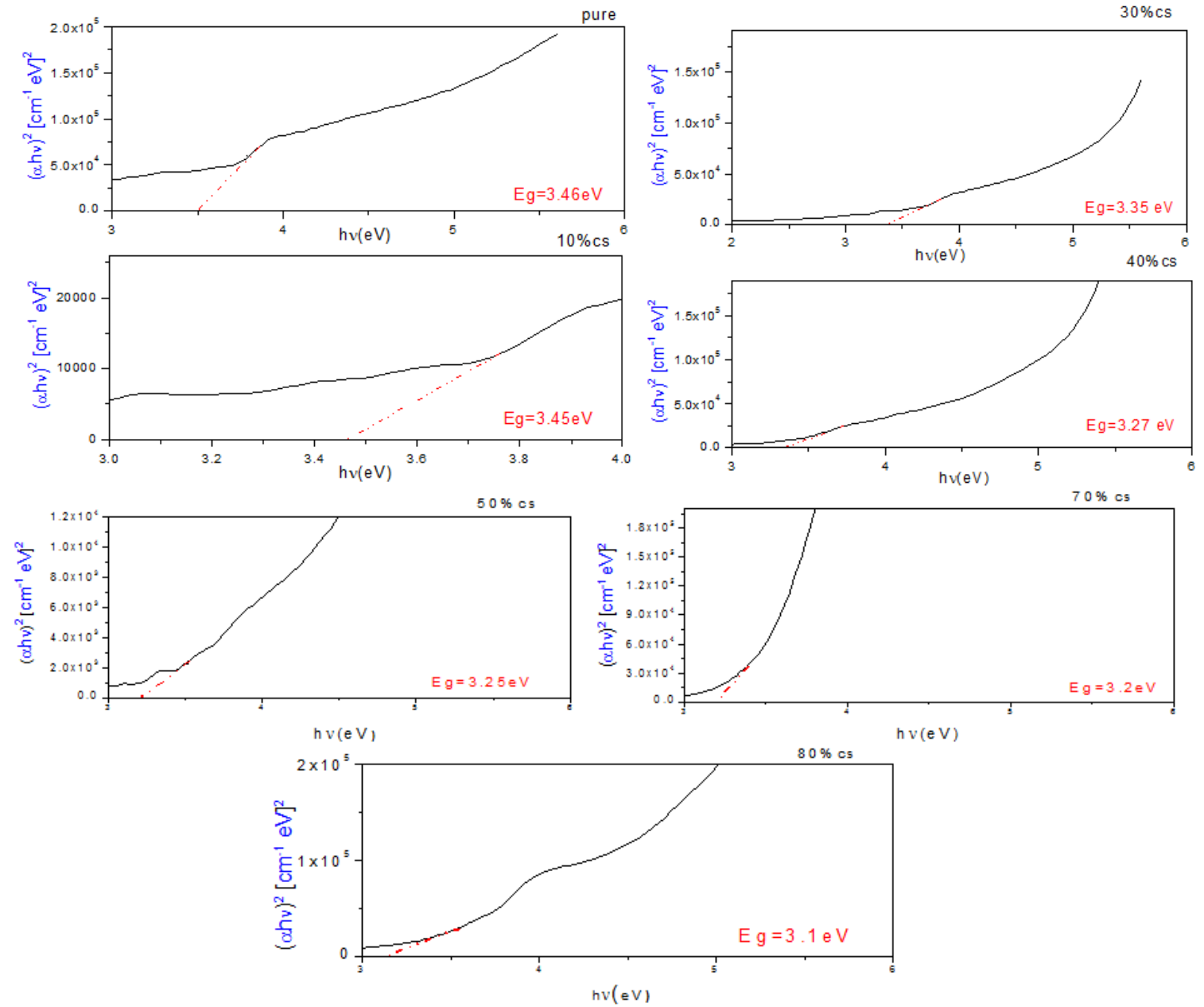

Fig 3: plots of $(\mathrm{ah} \cdot)^{2}$ as a function of photon energy $(\mathrm{h} \cdot$ ). 

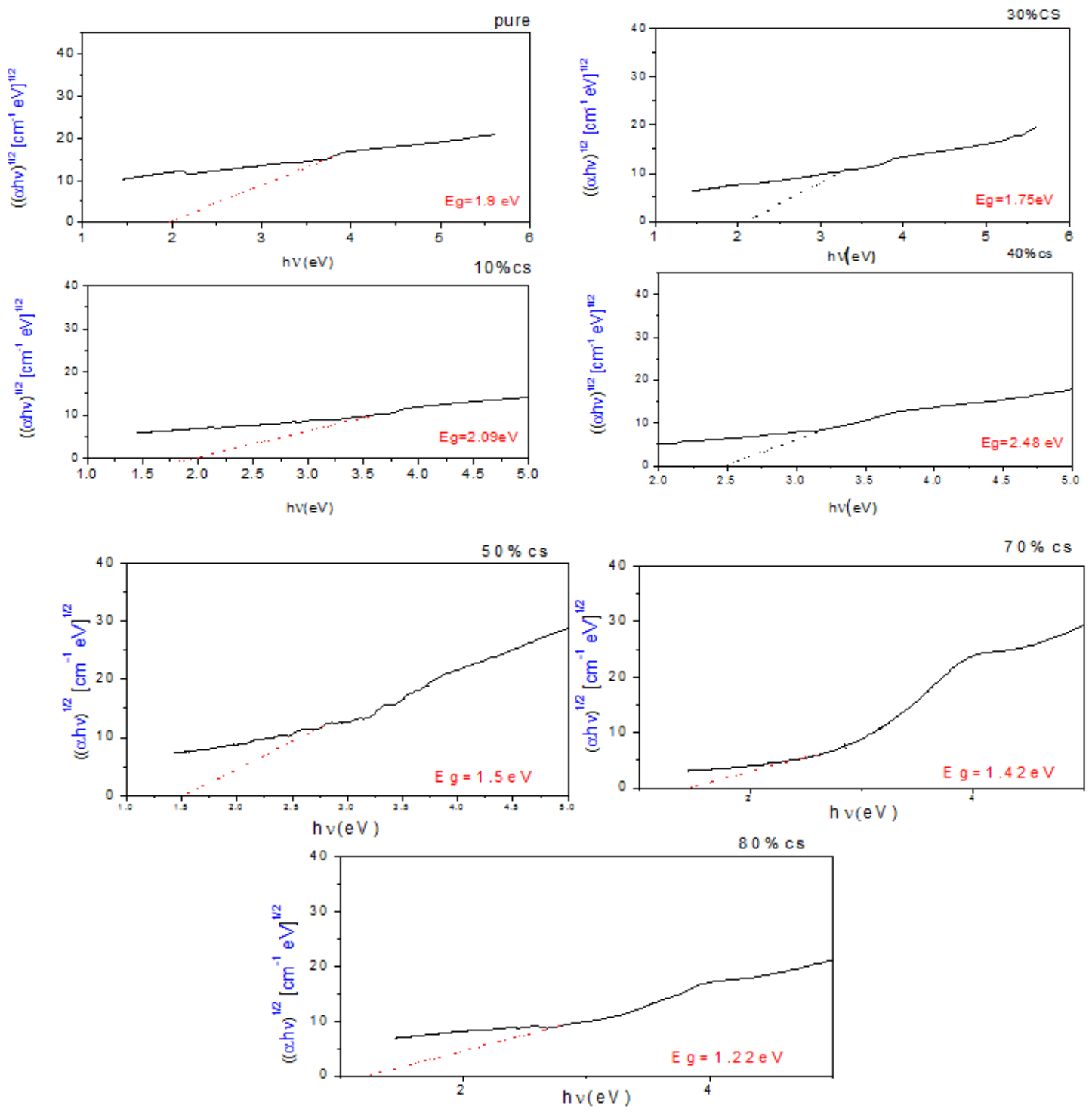

Fig 4: plot of $(a h v)^{1 / 2}$ as a function of photon energy $(h v)$. 

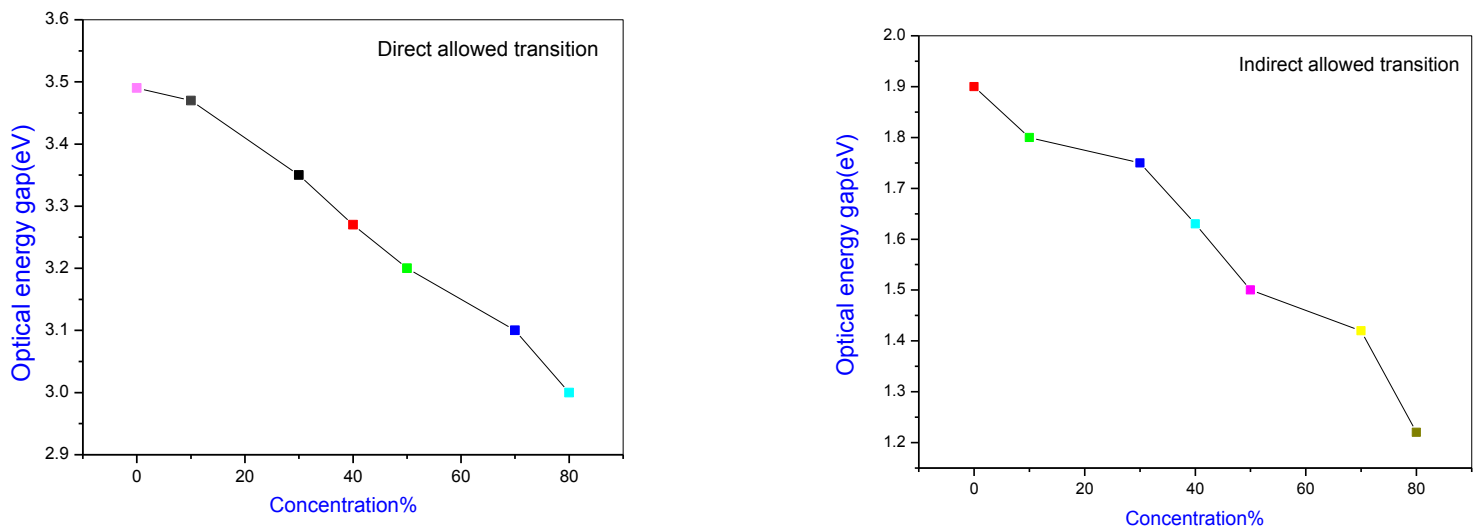

Fig 5: Calculated optical energy gap (Eg) for different chitosan content

\subsection{X-Ray Diffractograms}

X-ray diffraction pattern of prepared base blend PVA70/starch30 in addition to other samples containing different chitosan content were showed in Fig 6. It was clear that the main diffraction halos was observed nearly at $2 \theta=20^{\circ}$. Prepared poly-blend of (PVA/starch) was observed to be more crystalline than other films as verified through sharper main peak. Integration of various chitosan content to polymeric matrices result in a decrease in degree of crystallinity for all samples as a result of starch lost ordered arrangement during the gelatinisation route during chitosan addition as shown in Fig 6 .

The calculated area under the peak for these samples are shown in Table 2. The decrease in crystallinity is probably due to decreased intramolecular hydrogen bonding, and increased intermolecular hydrogen bonding with water.

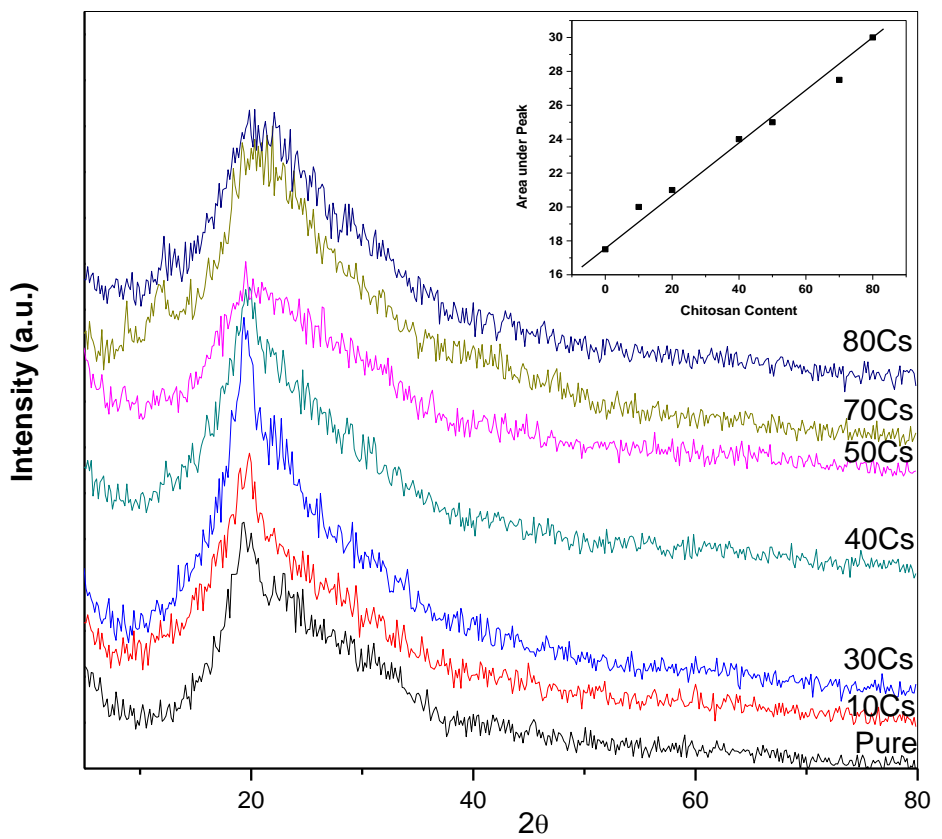

Fig 6: X-ray diffraction scans of PVA/Starch blend filled with different concentrations of Chitosan. 
Table 2. The area under of the peak of PVA/starch blend containing different concentration of chitosan from XRD

\begin{tabular}{|c|c|}
\hline PVA:Starch/Chitosan & Area under the peak \\
\hline $100 / 0$ & 17.5 \\
$90 / 10$ & 20 \\
$70 / 30$ & 21 \\
$60 / 40$ & 24 \\
$50 / 50$ & 25 \\
$30 / 70$ & 27.5 \\
$20 / 80$ & 30 \\
\hline
\end{tabular}

\subsection{Scanning Electron Microscopy (SEM)}

The morphologies of PVA/ starch blend and PVA/ starch containing chitosan films are shown in Fig 7. The surface of PVA/starch films is homogenous which is the sign of the structural integrity of the observed films.

SEM images of different chitosan content films revealed small granules and bright spots characteristic to starch. As the chitosan content increased, the starch phase changed from the dispersed phase, which indicates that amorphous starch is partially miscible with PVA [16].

Adding up to $80 \%$ more chitoan caused the cryo-fractured surface to become rougher and more brittle. This result shows an agreement with the XRD results.
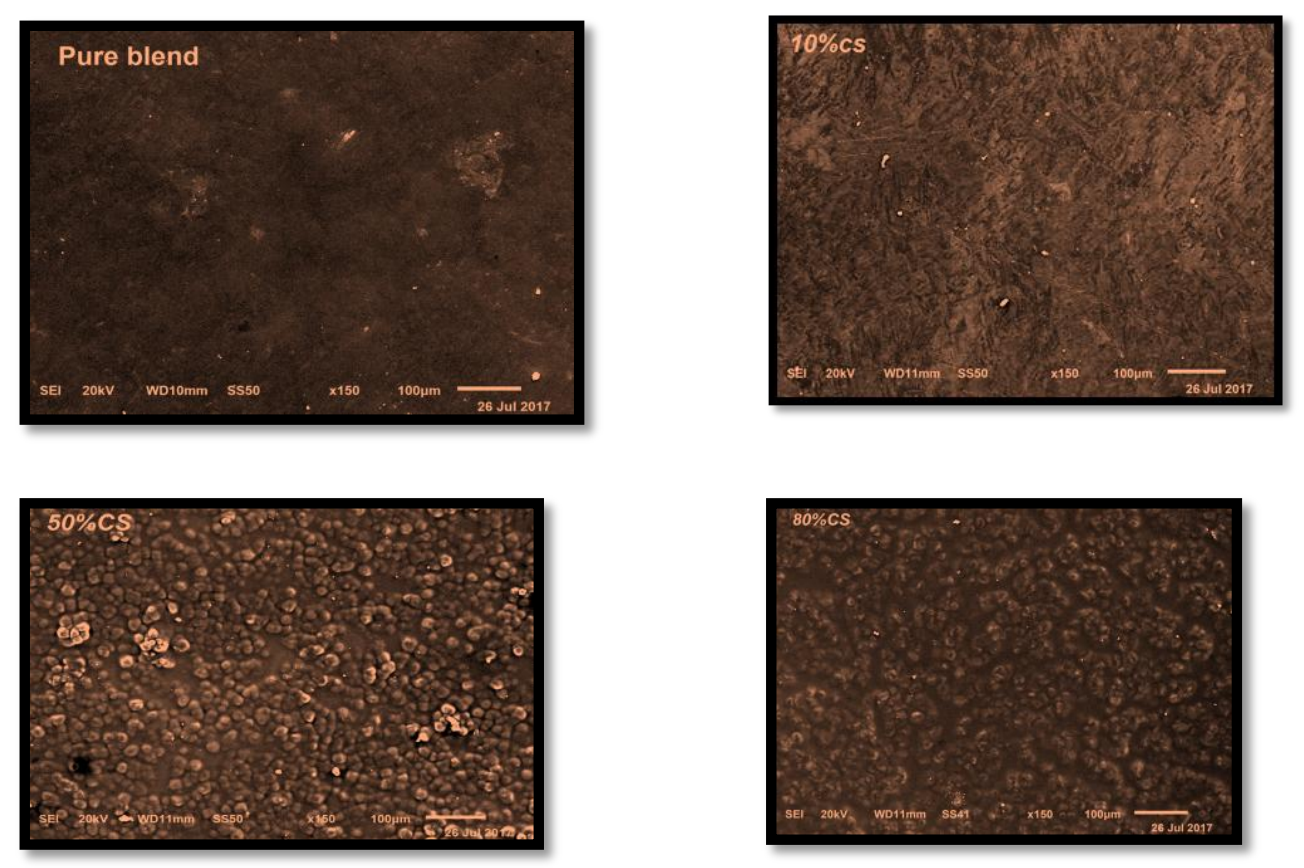

Fig 7: SEM surface morphology of PVA/starch blend containing different concentration of chitosan. 


\section{Conclusion}

The obtained results can be summarized as follows:

- $\quad$ FT-IR analysis delivers a vision for interaction possibilities between constituting partners.

- UV-vis examination displayed that, changing intensity and position of the observed shoulder around $308 \mathrm{~nm}$ characterize base blend (PVA/starch) indicate existence of interaction between chitosan and blend.

- XRD data exhibited that integration of chitosan into studied matrices causes observe decrease in the degree of crystallinity.

- SEM images revealed small granules and bright spots characteristic to starch. Adding up to $80 \%$ more chitoan caused the cryo-fractured surface to become rougher and more brittle. This shows an agreement with the XRD results.

- So the prepared films can be safely used in human contact applications.

\section{References}

[1] Kost J., Goldbart R.; (1997) Natural and Modified Polysaccharides In: Domb A. J., Kost J., Wiseman D. M. (eds), Handbook of Biodegradable Polymers. Hardwood Academic Publishers, Amsterdam, pp. 275289.

[2] Lu, D. R., C. M. Xiao, and S. J. Xu. "Starch-based completely biodegradable polymer materials." Express polymer letters 3, no. 6 (2009): 366-375.

[3] Kumar, Majeti NV Ravi. "A review of chitin and chitosan applications." Reactive and functional polymers 46, no. 1 (2000): 1-27.

[4] Khan, M. A., Z. Iqbal, M. Sarwar, M. Nisa, M. S. Khan, W. S. Lee, H. J. Lee, H. S. Kim, and K. S. Ki. "Urea treated corncobs ensiled with or without additives for buffaloes: Ruminal characteristics, digestibility and nitrogen metabolism." ASIAN AUSTRALASIAN JOURNAL OF ANIMAL SCIENCES 19, no. 5 (2006): 705.

[5] Wong, Dominic WS, Francois A. Gastineau, Kay S. Gregorski, Sandra J. Tillin, and Attila E. Pavlath. "Chitosan-lipid films: microstructure and surface energy." Journal of Agricultural and Food Chemistry 40, no. 4 (1992): 540-544.

[6] Crini, Gregorio, and Pierre-Marie Badot. "Application of chitosan, a natural aminopolysaccharide, for dye removal from aqueous solutions by adsorption processes using batch studies: A review of recent literature." Progress in polymer science 33, no. 4 (2008): 399-447

[7] Kumar, Majeti NV Ravi. "A review of chitin and chitosan applications." Reactive and functional polymers 46 , no. 1 (2000): 1-27.

[8] Rinaudo, Marguerite. "Chitin and chitosan: properties and applications." Progress in polymer science 31, no. 7 (2006): 603-632.

[9] Srinivasa, P. C., M. N. Ramesh, K. R. Kumar, and R. N. Tharanathan. "Properties and sorption studies of chitosan-polyvinyl alcohol blend films." Carbohydrate Polymers 53, no. 4 (2003): 431-438.

[10] Blout, Elkan R., and Robert Karplus. "The infrared spectrum of polyvinyl alcohol." Journal of The American Chemical Society 70, no. 2 (1948): 862-864. 
[11] Jayasekara, Ranjith, I. Harding, I. Bowater, G. B. Y. Christie, and Greg T. Lonergan. "Preparation, surface modification and characterisation of solution cast starch PVA blended films." Polymer testing 23, no. 1 (2004): 17-27.

[12] El-Kader, KAM Abd, SF Abdel Hamied, A. B. Mansour, A. M. Y. El-Lawindy, and F. El-Tantaway. "Effect of the molecular weights on the optical and mechanical properties of poly (vinyl alcohol) films." Polymer testing 21, no. 7 (2002): 847-850.

[13] Davis, P. W., and T. S. Shilliday. "Some optical properties of cadmium telluride." Physical Review 118, no. 4 (1960): 1020.

[14] Thutupalli, G. K. M., and S. G. Tomlin. "The optical properties of thin films of cadmium and zinc selenides and tellurides." Journal of Physics D: Applied Physics 9, no. 11 (1976): 1639.

[15] Shahada, Lamies, M. E. Kassem, H. I. Abdelkader, and H. M. Hassan. "Optical and electrical properties of a new polymer." Journal of applied polymer science 65, no. 9 (1997): 1653-1657.

[16] Zou, Guo-Xiang, Ping-Qu Jin, and Liang-Zou Xin. "Extruded starch/PVA composites: Water resistance, thermal properties, and morphology." Journal of Elastomers \& Plastics 40, no. 4 (2008): 303-316. 